\section{Biotinylated tags for recovery and characterization of ribonucleoprotein complexes}

\author{
Luiz O.F. Penalva and Jack D. Keene \\ Duke University Medical Center, Durham, NC, USA \\ BioTechniques 37:604-610 (October 2004)
}

Determining the in vivo targets of RNA-binding proteins and characterizing the posttranscriptional networks in which they participate constitute major challenges in the post-genomic era. An important step in this direction is the development of methods that permit efficient recovery of ribonucleoprotein (RNP) complexes. We present an improved methodology for efficient isolation of mammalian cell RNPS in which a biotin acceptor peptide $(B A P)$ is used to tag RNA-binding proteins. BAP-tagged RNA-binding proteins can be biotinylated in vivo by co-expression of the Escherichia coli BirA enzyme. RNP recovery was obtained using streptavidin sepharose beads, and messenger RNAs (mRNAs) were identified using multiprobe RNase protection assays and cDNA microarrays. Using this approach we efficiently recovered and quantified RNAs bound to cytoplasmic poly(A)-binding protein (PABP) and to nuclear human transformer 2 ( $h$ Tra-2) with minimal background.

\section{INTRODUCTION}

The implementation of genomebased platforms to characterize gene expression marked a new era in modern biology (reviewed in Reference 1). In the post-genomic era, there has been a major effort to characterize the gene expression profiles of different cells and tissues and also to investigate gene expression networks. Posttranscriptional regulation of gene expression plays a pivotal role in determining the quality and quantity of protein expression (2). RNA-binding proteins are the principal regulators of posttranscriptional events, which include pre-messenger RNA (pre-mRNA) splicing, mRNA transport and localization, and mRNA stability and translation (3). Using genomic technologies to characterize RNA subpopulations associated with specific RNA-binding proteins will help provide a better understanding of biological processes, including development and tumorigenesis (4).

Identifying the endogenous mRNA or pre-mRNA targets of RNA-binding proteins involved in different regulatory steps of mammalian cells has been challenging and has primarily been limited to in vitro-based methods (5-7). More recently, our laboratory and other laboratories have used in vivo meth- ods to immunoprecipitate and purify endogenous ribonucleoprotein (RNP) complexes and employed genomic technologies to identify RNA targets en masse in a quantitative manner (813). Although useful for determining RNA-binding protein targets, the current methodologies present technical challenges. For example, background signals and/or false positives that may be generated by nonspecific or, in some cases, specific binding of RNA to beads or antibodies (14), as well as inefficient RNA recovery because of low affinity antibodies. In order to address these and related issues, we designed an approach based on the use of small biotin acceptor peptides (BAP) to tag RNA-binding proteins and streptavidin-Sepharose ${ }^{\circledR}$ beads to purify the RNP complexes for subsequent gene expression profiling. The interaction between avidin and biotin is recognized for its high specificity and affinity, and as demonstrated in this study, it is highly advantageous for RNP and RNA recovery from cell extracts.

\section{MATERIALS AND METHODS}

\section{Cell Lines and Media}

293T cells were obtained from ATCC (Manassas, VA, USA) and main- tained in Dulbecco's modified Eagle medium (DMEM; Invitrogen, Carlsbad, Ca, USA) supplemented with $10 \%$ fetal bovine serum and antibiotics. Cells were plated in $150-\mathrm{mm}$ dishes coated with $0.001 \%$ poly-L-lysine (Sigma, St. Louis, MO, USA).

\section{Plasmid Construction}

BirA, the open reading frame (ORF) of the Escherichia coli BirA gene was cloned by PCR from E. coli genomic DNA and introduced into the pcDNA3 vector (Invitrogen). pSHREKK was generated by inserting into pcDNA3 a double-stranded oligonucleotide containing the sequence that encodes the peptide MSGLNDIFEAQKIEWHE. This sequence is based on the BAP \#85 (15). BAP-poly(A)-binding protein (PABP) was prepared by inserting the sequence encoding the peptide recognized by the tobacco etch virus (TEV) protease (ENLYFQG) followed by the poly(A)-binding protein I (PABP-I) ORF into pSHREKK (upstream BAP). BAP-human transformer 2 (hTra-2) was constructed identically to the BAPPABP clone but using the human transformer- $2 \beta$ isoform ORF included in place of the PABP-I sequence. PEGFPN1 (BD Biosciences Clontech, Palo Alto, CA, USA) was used as a control.

\section{Transient Transfection of 293T Cells}

Eight micrograms DNA were used to transfect $293 \mathrm{~T}$ cells plated as described above. At the time of transfection, cells were typically 50\%-60\% confluent. Transfections were carried out following the conditions described in GeneJammer ${ }^{\mathrm{TM}}$ (Stratagene, La Jolla, CA, USA) instruction booklet. Biotin (Sigma) was added to the medium at the time of transfection.

\section{Western Blot Analysis}

Fifty micrograms extract were fractionated by electrophoresis in 4\%-20\% polyacrylamide sodium dodecyl sulfate (SDS) Laemmli gels. Proteins were transferred to nitrocellulose membranes using a transfer cell (Bio-Rad Laboratories, Hercules, CA, USA). After blocking with $5 \%$ nonfat milk and $0.1 \%$ Tween ${ }^{\circledR} 20$ in phosphate-buffered sa- 
line (PBS) buffer, the membranes were incubated with 1:1000 diluted streptavidin conjugated with horseradish peroxidase (HRP; Chemicon, Temecula, CA, USA). Alternatively, the membranes were incubated with anti-PABP rabbit serum (1:1000 dilution). Antirabbit HRP immunoglobulin G (IgG; Amersham Biosciences, Piscataway, NJ, USA) was used as secondary antibody at a 1:10,000 dilution. Blots were washed with PBS- $0.1 \%$ Tween 20 , developed using electrochemiluminescent $\left(\mathrm{ECL}^{\mathrm{TM}}\right)$ detection kits (Amersham Biosciences), and exposed to film.

\section{Preparation of Cell Lysates from 293T Transfected Cells}

Forty-eight hours after transfection, cells were washed twice with ice-cold PBS, harvested using a scraper, and pelleted by centrifugation at 1500 $2000 \times g$ for $5 \mathrm{~min}$ at $4^{\circ} \mathrm{C}$. The cell pellet was resuspended and homogenized in 1.5 volumes of lysis buffer [ $100 \mathrm{mM}$ $\mathrm{KCl}, 25 \mathrm{mM}$ EDTA, $5 \mathrm{mM} \mathrm{MgCl}_{2}, 10$ mM HEPES, pH 7.0, 0.5\% Nonidet ${ }^{\circledR}$ P-40 (NP40), 10\% glycerol, $2 \mathrm{mM}$ dithiotreitol (DTT), $0.4 \mathrm{mM}$ vanadyl ribonucleoside complex (VRCs; New England Biolabs, Beverly, MA, USA), 1 tablet of Complete Proteinase Inhibitor (Roche, Basel, Switzerland) $/ 50 \mathrm{~mL}$ buffer, and $100 \mathrm{U} / \mathrm{mL}$ RNaseOUT ${ }^{\mathrm{TM}}$ (Invitrogen)]. The lysate was centrifuged at $13,000 \times g$ in a microfuge for $10 \mathrm{~min}$ at $4^{\circ} \mathrm{C}$. The supernatant was transferred to a new tube, and the pel- let was treated as before, but this time using only one volume of lysis buffer. The supernatants were combined and stored at $-80^{\circ} \mathrm{C}$ in aliquots of $400 \mu \mathrm{L}$ (16). $293 \mathrm{~T}$ cell lysates produced by this method typically range in concentration from $15-20 \mathrm{mg} / \mathrm{mL}$ of total protein.

\section{RNP Purification and RNA Preparation}

The 400- $\mu \mathrm{L}$ lysate samples were centrifuged in a microfuge at $13,000 \times g$ for $10 \mathrm{~min}$ at $4^{\circ} \mathrm{C}$. The supernatants were transferred to new tubes containing 10 volumes of NT2 buffer (50 mM Tris, pH 7.4, $150 \mathrm{mM} \mathrm{NaCl}$, $1 \mathrm{mM} \mathrm{MgCl}, 0.05 \% \mathrm{NP} 40$ ) plus 25 $\mathrm{mM}$ EDTA, $2 \mathrm{mM}$ DTT, $100 \mathrm{U} / \mathrm{mL}$ RNaseOut, and $0.4 \mathrm{mM}$ VRC. Packed streptavidin-Sepharose beads (125-150 $\mu \mathrm{L}$; Amersham Biosciences) were resuspended in 10 volumes of NT2 buffer supplemented with $5 \%$ bovine serum albumin (BSA) and incubated for 30 min at $4^{\circ} \mathrm{C}$. Beads were washed three times with 10 volumes of NT2 buffer and subsequently added to the diluted lysates. Precipitation reactions were tumbled end-over-end at room temperature for $3 \mathrm{~h}$. Following the incubation, the beads were centrifuged for $2 \mathrm{~min}$ at $1000-1500 \times g$ at $4^{\circ} \mathrm{C}$ and washed with approximately 10 bead volumes of icecold NT2 buffer. This operation was repeated four more times. The beads were then resuspended in $400 \mu \mathrm{L}$ of $1 \times$ TEV protease buffer containing $40 \mathrm{U}$ TEV protease (Invitrogen). The diges-

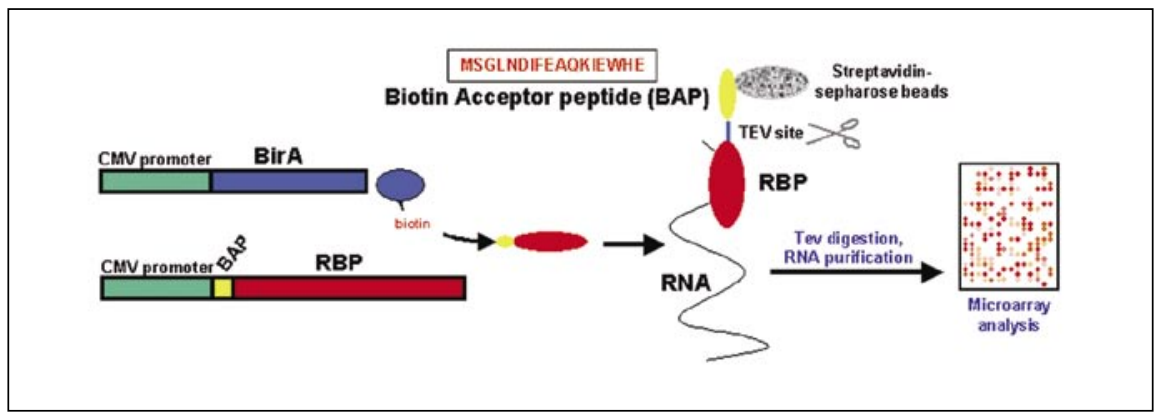

Figure 1. Strategy used to characterize in vivo targets of RNA-binding proteins using biotinylation in vivo. Cells are cotransfected with a construct expressing an RNA-binding protein tagged with a biotin acceptor peptide (BAP) and a construct expressing the Escherichia coli BirA gene. Biotinylation of the tag occurs only in the presence of the BirA enzyme. Specific ribonucleoprotein (RNP) complexes are purified by centrifugation using avidin or streptavidin-Sepharose beads. A tobacco etch virus (TEV) protease recognition site is placed between the tag and the RNA-binding protein sequence to further decrease background. Digestion with TEV protease releases the RNA-binding protein and its associated RNA targets. The RNA samples are phenol-extracted, precipitated, and subsequently analyzed by RNase protection assays (RPAs) or microarrays.

tions were performed in a hybridization oven for $2 \mathrm{~h}$ at $30^{\circ} \mathrm{C}$. After incubation, $400 \mu \mathrm{L}$ of $2 \times$ proteinase $\mathrm{K}$ digestion mixture (100 mM Tris, pH 7.5, $10 \mathrm{mM}$ EDTA, $50 \mathrm{mM} \mathrm{NaCl}, 1 \%$ SDS, $500 \mu \mathrm{g}$ proteinase $\mathrm{K}$ ) were added to the tubes. Reactions were placed back into the hybridization oven and tumbled for additional $30 \mathrm{~min}$ at $50^{\circ} \mathrm{C}$. Beads were centrifuged at $1500-2000 \times g$ for $3 \mathrm{~min}$ at $4^{\circ} \mathrm{C}$ and the supernatants were transferred to new tubes containing $700 \mu \mathrm{L}$ of phenol-chloroform. Samples were vortex mixed for $30 \mathrm{~s}$ and centrifuged at $13,000 \times g$ for $5 \mathrm{~min}$ at $4^{\circ} \mathrm{C}$. The extraction was repeated with one volume of chloroform. RNA was precipitated by the addition of 0.8 volumes of isopropanol, $80 \mu \mathrm{L} 4 \mathrm{M}$ ammonium acetate, 3 $\mu \mathrm{L} 1 \mathrm{M} \mathrm{MgCl}_{2}$, and $8 \mu \mathrm{L}$ glycogen 20 $\mathrm{mg} / \mathrm{mL}$ (Roche). Samples were stored at $-80^{\circ} \mathrm{C}$ until ready for gene expression analysis. RNA was recovered by centrifugation $(13,000 \times g$ for $30 \mathrm{~min}$ at $4^{\circ} \mathrm{C}$ ), washed with $100 \mu \mathrm{L}$ of $80 \%$ ethanol, and resuspended in water or hybridization buffer depending on the gene expression analysis method that will be performed.

\section{Gene Expression Analysis}

RNase protection assays (RPAs). Precipitated RNAs were assayed by RNase protection using the RiboQuant $^{\mathrm{TM}}$ assay (BD Biosciences Pharmingen, San Diego, CA, USA) according to the manufacturer's recommendations (45014K). The human tumor suppressor (hTS1) template set (556161) was used in the experiment shown here. Protected riboprobe fragments were visualized on a phosphorimaging screen. Phosphorimages were scanned using the Storm ${ }^{\text {TM }} 860$ system (Amersham Biosciences) at $100 \mu \mathrm{m}$ resolution and analyzed by using ImageQuant $^{\mathrm{TM}}$ software (ver. 5.0).

Atlas $^{\mathrm{TM}}$ microarrays. cDNA array analysis was performed by using Atlas Human 1.2 Arrays (BD Biosciences Clontech). Probing of cDNA arrays was performed as described in the Atlas cDNA Expression Arrays user's manual. Reverse-transcribed probes were radiolabeled using $\left[\alpha-{ }^{32} \mathrm{P}\right] \mathrm{dATP}$ (Amersham Biosciences). After hybridization, each array membrane was washed, and the results were visualized by phospho- 
rimaging as described above. Images were analyzed using Atlas Image 2.01 software (BD Biosciences Clontech).

\section{RESULTS AND DISCUSSION}

BAPs have been used as protein tags in vitro and in vivo in both prokaryotic and eukaryotic systems for purification of proteins and macromolecular complexes (15,17-19). BAPs constitute a powerful alternative to current antibody epitope tags because of their high affinity and reproducibility. Since no antibodies are required, and streptavidin-Sepharose beads are relatively inexpensive compared with protein A or G Sepharose beads, the experimental costs are dramatically reduced. Here, we explore the use of biotinylated tags as a tool to recover and characterize RNP complexes.

To demonstrate the effectiveness and flexibility of this method, we chose a cytoplasmic RNA-binding protein (PABP I) and a nuclear protein (hTra-2), which play roles in translation and splicing, respectively $(20,21)$. The presence of the BAP tags did not affect cellular localization of PABP and hTra-2 in comparison to wild-type proteins (data not shown).

Figure 1 represents a schematic view of this methodology. 293T cells were transiently cotransfected with constructs expressing BAP-tagged RNA-binding proteins and the E. coli BirA enzyme, which catalyzes the biotinylation process in vivo (19). Cell extracts were prepared $48 \mathrm{~h}$ posttransfection. The RNP complexes are then purified by precipitation with streptavidin-Sepharose beads. As part of the strategy to decrease the background due to nonspecific binding of RNA to the beads, we introduced a TEV NIa protease recognition sequence between the RNA-binding protein and the BAP tag (22). After precipitation, the beads were washed, resuspended in protease buffer, and digested with TEV, thereby releasing the RNPs into the supernatant. The beads were centrifuged and discarded. RNAs are extracted, precipitated, and analyzed using microarrays or RNase protection assays. See Materials and Methods for a more detailed description.
We initially assessed by Western blot analysis the specificity and efficiency of the biotinylation reaction. The major concern was the biotinylation of other endogenous RNA-binding proteins that could present a problem by generating false positives. No biotinylated products other than the transfected BAP-RNA-binding proteins were detected under these conditions, and as expected, both
BAP-PABP and BAP-hTra-2 were biotinylated only when the BirA product was co-expressed (Figure 2, A and B). Moreover, biotinylation was dramatically enhanced when additional biotin (100 or $1000 \mu \mathrm{M}$ ) was added to the medium. Compare Figure 2A lane 3 (no added biotin) to lane $4(100 \mu \mathrm{M}$ biotin) and lane 5 (1000 $\mu \mathrm{M}$ biotin).

We next tested the efficiency of biotinylation by binding BAP-PABP in

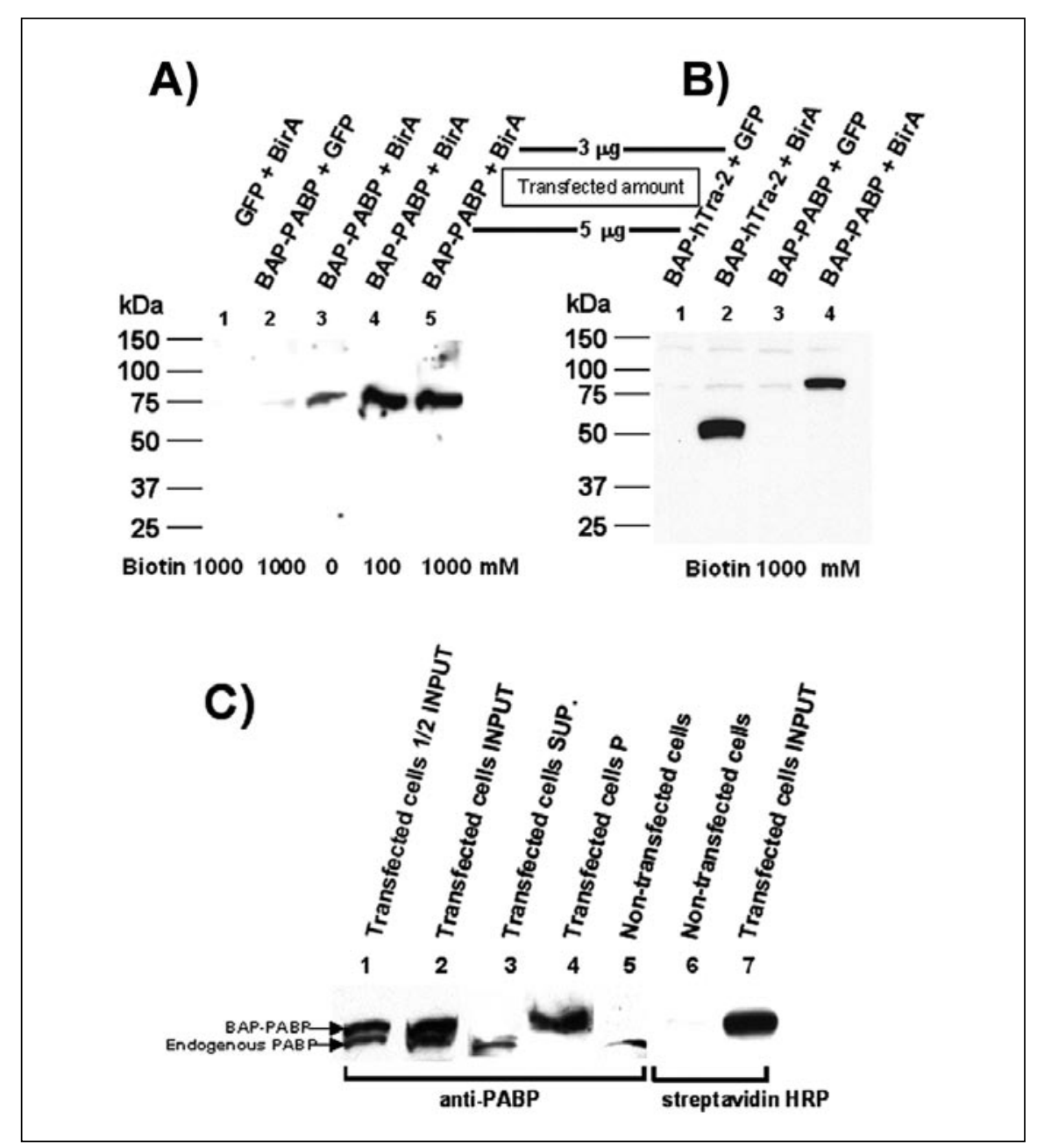

Figure 2. Western blot analysis of 293T cells transfected with biotin acceptor peptide (BAP)-tagged RNA-binding proteins. (A) Cotransfections using BAP- poly(A)-binding protein (PABP), BirA, or green fluorescent protein (GFP) demonstrated that: $(i)$ biotinylation of BAP-PABP occurs in vivo only in the presence of the Escherichia coli BirA enzyme; (ii) no other biotinylated proteins were detected in these 293T cell extracts; and (iii) addition of increasing amounts of biotin (100 or $1000 \mu \mathrm{M})$ to the growth medium increased the extent of biotinylation of BAP-PABP. Western blot analyses were probed with avidin conjugated to horseradish peroxidase (HRP). (B) Biotinylation of BAP-human transformer 2 (hTra2) also occurred only in the presence of BirA enzyme. (C) Immunoprecipitation followed by Western blot analysis of $293 \mathrm{~T}$ cells transfected with BAP-PABP shows high efficiency of biotinylation. $293 \mathrm{~T}$ cell extract containing approximately $50 \mu \mathrm{g}$ of total protein was precipitated with $10 \mu \mathrm{L}$ of packed streptavidin-Sepharose beads. Western blot analyses probed with anti-PABP antibody showed both the endogenous PABP and the BAP-PABP (lane 1). Extracts from nontransfected cells were used as a reference for the endogenous PABP (lane 5). A Western blot analysis probed with streptavidin-HRP was used as a reference marker for the BAP-PABP. Comparison of the material eluted from the beads (lane 4) with the supernatant (lane 3 ) and input (lane 2) shows the efficiency of biotinylation and protein recovery. 


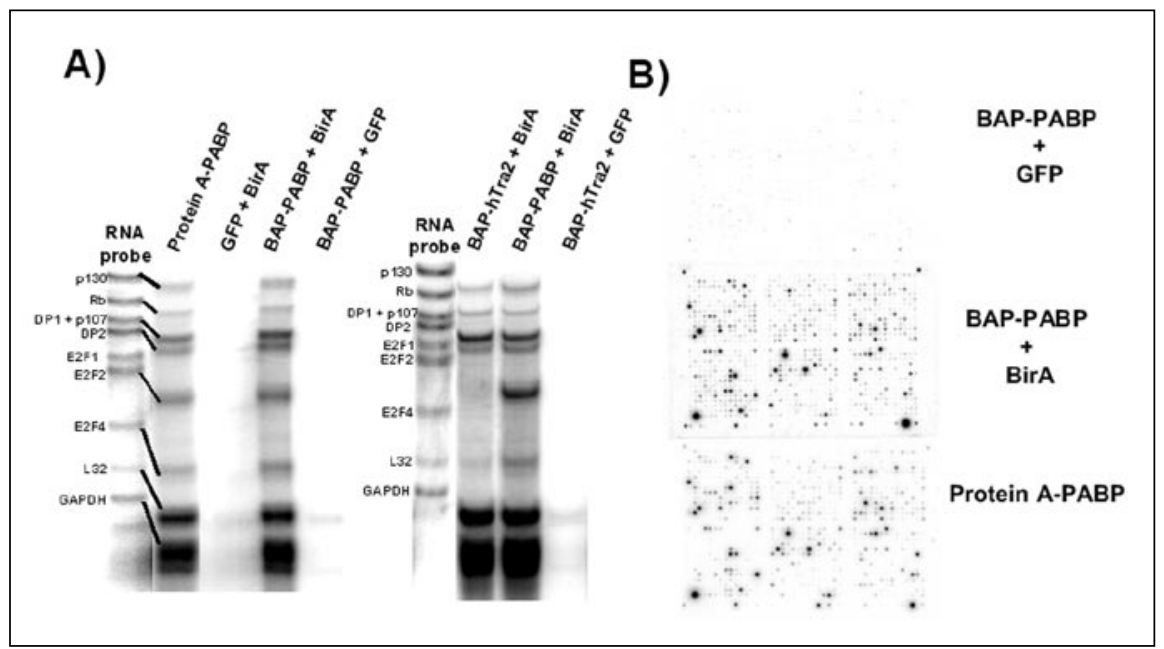

Figure 3. Gene expression analysis of 293T cells transfected with biotin acceptor peptide (BAP)tagged RNA-binding proteins. Cell lysates $(400 \mu \mathrm{L} ; 15-20 \mathrm{mg} / \mathrm{mL})$ from separate transfection experiments were precipitated with streptavidin-Sepharose beads. A transfection experiment with protein A-tagged poly(A)-binding protein (PABP) was used as a positive control. In this case, Sepharose immunoglobulin $\mathrm{G}(\mathrm{IgG})$ beads were used to recover the ribonucleoprotein (RNP) complexes. RNA samples obtained were analyzed by: (A) RNase protection assay (RPA) with a human tumor suppressor 1 probe set (Invitrogen) and unprotected RNA probes used to identify the protected fragments of different size and (B) Human 1.2 Atlas arrays used for comparison between BAP - PABP + BirA and BAP - PABP + green fluorescent protein (GFP) arrays (detailed data provided in the supplementary material found on the BioTechniques' web site at http://BioTechniques.com/Oct04/PenalvaSupplementary.html). Results show that: (i) RNA can be recovered and quantified only when the BAP-tagged RNA-binding proteins [PABP and human transformer 2 (hTra-2)] are co-expressed with the BirA enzyme; (ii) when BirA was substituted with GFP, no RNA could be detected; and (iii) no RNA spots were apparent on microarrays when GFP was used in place of the RNA-binding proteins, an indication that biotinylation of other endogenous RNA-binding proteins was not detectable.

293T extracts to streptavidin-Sepharose beads (Figure 2C). Nonbiotinylated BAP-PABP should not bind steptavidin and consequently is expected to remain in the supernatant. Comparison of the material eluted from the beads (lane 4) to the supernatant (lane 3) and to the input (lane 2) showed that almost all BAP-PABP was biotinylated and that it could also be efficiently recovered using streptavidin-Sepharose beads. Similar recoveries were reported previously using a mammalian transcription factor (19).

In order to evaluate the efficiency of RNA recovery, specificity and background using this methodology, we cotransfected 293T cells with either the BAP-PABP or BAP-hTra2 constructs in combination with constructs encoding either the BirA enzyme or control green fluorescent protein (GFP). Cell extracts and precipitations were performed as described above. RNA samples were analyzed by both multiprobe RPAs (Figure 3A) and Atlas cDNA microarrays (Figure 3B). Protein A-tagged PABP was used as a positive control. As can be seen in Figure 3, mRNAs were recovered and detected only when the BirA enzyme was co-expressed with the tagged RNA-binding protein. Minimal background was observed when GFP was substituted in place of BirA. Moreover, when the BAP-RNA-binding protein was substituted with control GFP, no RNA products were detected (Figure 3A and data not shown). This result suggests that if other endogenous RNA-binding proteins were biotinylated, any associated mRNAs represented in the probe set were below background detection. A detailed analysis of the microarray data shown in Figure 3B (BAP - $\mathrm{PABP}+\mathrm{BirA} \times \mathrm{BAB}-\mathrm{PABP}+\mathrm{GFP})$ is provided as supplementary material found on the BioTechniques' web site at http://BioTechniques.com/Oct04/ PenalvaSupplementary.html.

In conclusion, the methodology described here provides a high affinity alternative for biochemical characterization of RNP complexes and can be used with animal models for gene expression profiling of specific cell types in tissues or tumors $(4,7,8)$.

\section{ACKNOWLEDGMENTS}

The authors would like to thank Diego Zorio, Mike Burdick, Dale Beach, and Amy Sims for comments on the manuscript and Dhanrajan Tiruchinapalli for helping with the figures.

\section{COMPETING INTERESTS STATEMENT}

The authors declare no conflicts of interest.

\section{REFERENCES}

1.Lockhart, D.J. and E.A. Winzeler. 2000. Genomics, gene expression and DNA arrays. Nature 405:827-836.

2.Keene, J.D. and S.A. Tenenbaum. 2002. Eukaryotic mRNPs may represent posttranscriptional operons. Mol. Cell 9:1161-1167.

3.Siomi, H. and G. Dreyfuss. 1997. RNA-binding proteins as regulators of gene expression. Curr. Opin. Genet. Dev. 7:345-353.

4.Keene, J.D. 2001. Ribonucleoprotein infrastructure regulating the flow of genetic information between the genome and the proteome. Proc. Natl. Acad. Sci. USA 98:7018-7024.

5.Tsai, D.E., D.S. Harper, and J.D. Keene. 1991. U1-snRNP-A protein selects a ten nucleotide consensus sequence from a degenerate RNA pool presented in various structural contexts. Nucleic Acids Res. 19:4931-4936.

6.Tuerk, C. and L. Gold. 1990. Systematic evolution of ligands by exponential enrichment: RNA ligands to bacteriophage T4 DNA polymerase. Science 249:505-510.

7.Gao, F., C. Carson, T.D. Levine, and J.D. Keene. 1994. Selection of a subset of mRNAs from $3^{\prime}$ UTR combinatorial libraries using neuronal RNA-binding protein, Hel-N1. Proc. Natl. Acad. Sci. USA 91:11207-11211.

8.Tenenbaum, S.A., C.C. Carson, P.J. Lager, and J.D. Keene. 2000. Identifying mRNA subsets in messenger ribonucleoprotein complexes by using cDNA arrays. Proc. Natl. Acad. Sci. USA 97:14085-14090.

9.Brown, V., P. Jin, S. Ceman, J.C. Darnell, W.T. O'Donnell, S.A. Tenenbaum, X. Jin, Y. Feng, et al. 2001. Microarray identification of FMRP-associated brain mRNAs and altered mRNA translational profiles in fragile $\mathrm{X}$ syndrome. Cell 107:477-487.

10.Waggoner, S.A. and S.A. Liebhaber. 2003. Identification of mRNAs associated with alphaCP2-containing RNP complexes. Mol. Cell. Biol. 23:7055-7067.

11.Liu, W., J. Seto, E. Sibille, and M. Toth. 2003. The RNA binding domain of Jerky consists of tandemly arranged helix-turn-helix/ homeodomain-like motifs and binds specific sets of mRNAs. Mol. Cell. Biol. 23:40834093.

12.Eystathioy, T., E.K.L. Chan, S.A. Tenenbaum, J.D. Keene, K. Griffith, and M.J. 
Fritzler. 2002. A phosphorylated cytoplasmic autoantigen, GW182, associates with a unique population of human mRNAs within novel cytoplasmic speckles. Mol. Biol. Cell 13:1338-1351.

13.Ule, J., K.B. Jensen, M. Ruggiu, A. Mele, A. Ule, and R.B. Darnell. 2003. CLIP identifies Nova-regulated RNA networks in the brain. Science 302:1212-1215.

14.Lipes, B.D. and J.D. Keene. 2002. Autoimmune epitopes in messenger RNA. RNA 8:762-771.

15.Schatz, P.J. 1993. Use of peptide libraries to map the substrate specificity of a peptidemodifying enzyme: a 13 residue consensus peptide specifies biotinylation in Escherichia coli. Biotechnology 11:1138-1143.

16.Penalva, L.O.F., S.A. Tenenbaum, and J.D. Keene. 2004. Gene expression analysis of messenger RNP complexes. Methods Mol. Biol. 257:125-134.

17.Cull, M.G. and P.J. Schatz. 2000. Biotinylation of proteins in vivo and in vitro using small peptide tags. Methods Enzymol. 326:430-440.

18.Parrott, M.B. and M.A. Barry. 2000. Metabolic biotinylation of recombinant proteins in mammalian cells and in mice. Mol. Ther. 1:96-104.

19.de Boer, E., P. Rodriguez, E. Bonte, J. Krijgsveld, E. Katsantoni, A. Heck, F. Grosveld, and J. Strouboulis. 2003. Efficient biotinylation and single-step purification of tagged transcription factors in mammalian cells and transgenic mice. Proc. Natl. Acad. Sci. USA 100:7480-7485.

20.Kahvejian, A., G. Roy, and N. Sonenberg. 2001. The mRNA closed-loop model: the function of PABP and PABP-interacting proteins in mRNA translation. Cold Spring Harb. Symp. Quant. Biol. 66:293-300.

21.Tacke, R., M. Tohyama, S. Ogawa, and J.L. Manley. 1998. Human Tra2 proteins are sequence-specific activators of pre-mRNA splicing. Cell 93:139-148.

22.Dougherty, W.G., J.C. Carrington, S.M. Cary, and T.D. Parks. 1988. Biochemical and mutational analysis of a plant virus polyprotein cleavage site. EMBO J. 7:1281-1287.

Received 10 May 2004; accepted 29 June 2004.

Address correspondence to Jack D. Keene, Duke University Medical Center, Center for RNA Biology, Department of Molecular Genetics \& Microbiology, Box 3020, Durham, NC 27710,USA.e-mail: keene001@mc. duke.edu

\title{
Real-time quantitative PCR-based system for determining transgene copy number in transgenic animals
}

\author{
Maria Ballester, Anna Castelló, Elena Ibáñez, Armand Sánchez, \\ and Josep M. Folch \\ Universitat Autònoma de Barcelona, Bellaterra, Spain
}

BioTechniques 37:610-613 (October 2004)

In this paper, we describe a rapid and accurate real-time quantitative PCR-based system to determine transgene copy number in transgenic animals. We used the $2^{-\Delta \Delta C t}$ method to analyze different transgenic lines without the requirement of a control sample previously determined by Southern blot analysis. To determine the transgene copy number in several mouse lines carrying a goat $\beta$-Lactoglobulin transgene, we developed a TaqMan ${ }^{\circledR}$ assay in which a goat genomic DNA sample was used as a calibrator. Moreover, we used the glucagon gene as a reference control because this gene is highly conserved between species and amplifies with the same efficiency and sensitivity in goat as in mouse. With this assay, we provide an alternative simple method to determine the transgene copy number, avoiding the traditional and tedious blotting techniques. The assay's discrimination ability from our results is of at least six copies and, similar to the limitations of the blotting techniques, the accuracy of the quantification diminishes when the transgene copy number is high.

\section{INTRODUCTION}

The pronuclear microinjection technique has been used as an important tool for the production of transgenic animals. However, this procedure is characterized by random integration of the transgene, usually as multiple copies in tandem that vary in number between transgenic lines (1). Traditionally, the transgene copy number in both founders and G1 hemizygous transgenic mice has been analyzed by blotting techniques (i.e., Southern blot analysis, dot blot, and slot blot), which are tedious and time-consuming methods that require a large amount of DNA sample for each assay. Moreover, the quantification by those methods is not accurate and gives ambiguous results.

With the emergence of the real-time quantitative PCR technology, different applications have been described for the analysis of transgenic organisms, such as the determination of transgene copy number in transformed plants (2) or the study of zygosity in transgenic animals $(3,4)$.

Here we present a new application of the real-time quantitative PCR for transgene copy number determination in transgenic animals, characterized by the speed, high-throughput, sensitivity, and accuracy of the TaqMan ${ }^{\circledR}$ methodology $(5,6)$. For this purpose, we used the $2^{-\Delta \Delta C t}$ method (7) to compare the $\Delta \mathrm{C}_{\mathrm{t}}$ [cycle threshold $\left(\mathrm{C}_{t}\right) ; \mathrm{C}_{t}$ of target minus $\mathrm{C}_{t}$ of control gene] value of transgenic animal samples with unknown transgene copy numbers with the $\Delta \mathrm{Ct}$ of a known calibrator. Unlike the approach used by Tesson et al. (3), the use of a control sample previously analyzed by Southern blot analysis is not required in the current method. A goat genomic DNA sample, carrying a single copy of the $\beta$-Lactoglobulin $(\beta L G)$ gene, was used as a calibrator to determine the transgene copy number in several caprine $\beta L G$ transgenic mice lines. Moreover, we describe the primers and TaqMan probe design that amplify with the same efficiency and sensitivity a highly conserved sequence of the glucagon gene in goat as in mouse samples that can be used for endogenous control gene amplification in both species.

\section{MATERIALS AND METHODS}

\section{Animals and DNA Samples}

Transgenic mice were previously generated by pronuclear microinjection of the pPX(7.0) construct, which con- 